This item was submitted to Loughborough's Research Repository by the author.

Items in Figshare are protected by copyright, with all rights reserved, unless otherwise indicated.

\title{
Performance characterisation of photovoltaic modules
}

PLEASE CITE THE PUBLISHED VERSION

http://dx.doi.org/10.1109/PVSC.2010.5614204

PUBLISHER

(c) IEEE

VERSION

VoR (Version of Record)

LICENCE

CC BY-NC-ND 4.0

REPOSITORY RECORD

Gottschalg, Ralph. 2019. "Performance Characterisation of Photovoltaic Modules". figshare. https://hdl.handle.net/2134/7914. 
This item was submitted to Loughborough's Institutional Repository (https://dspace.lboro.ac.uk/) by the author and is made available under the following Creative Commons Licence conditions.

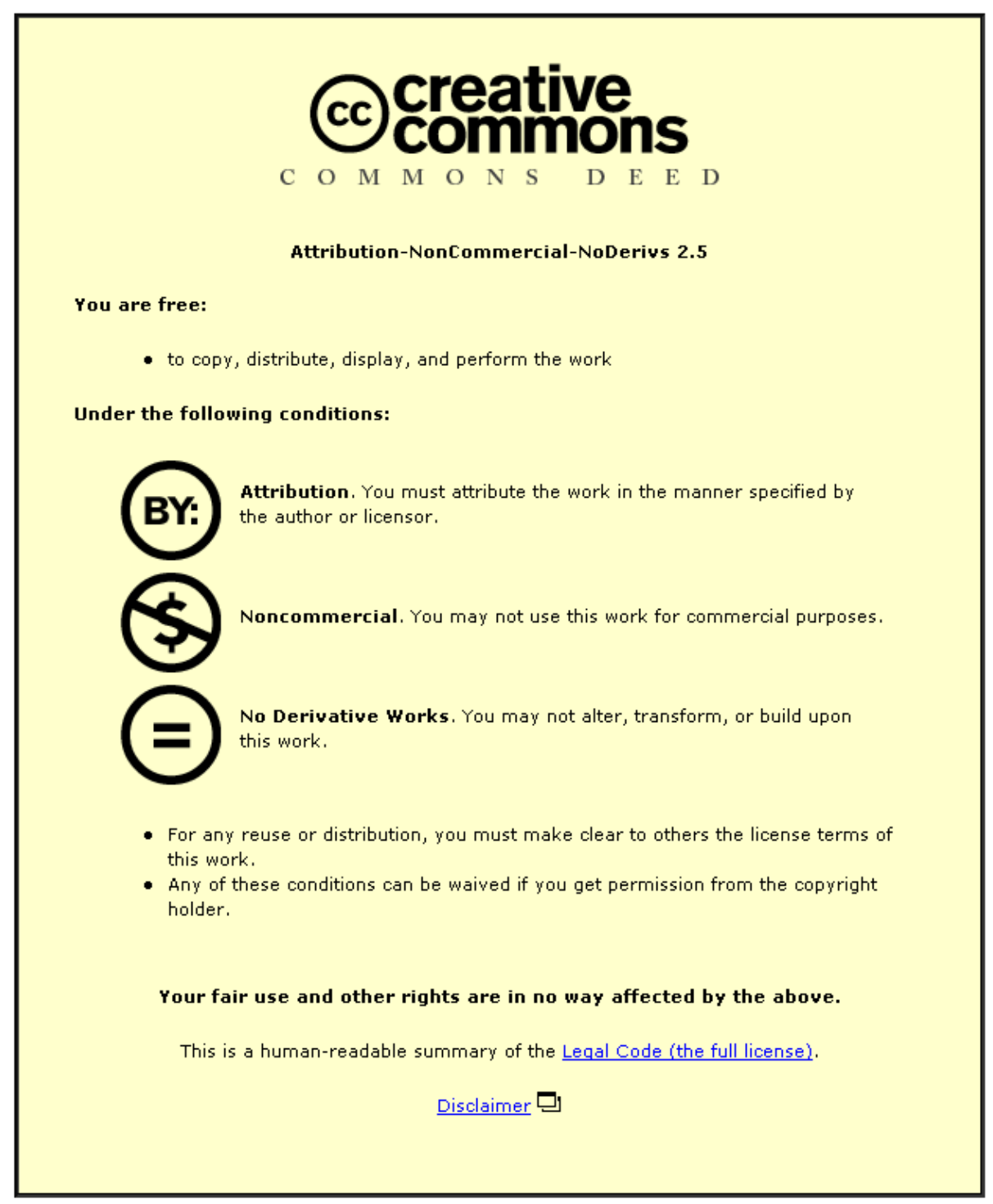

For the full text of this licence, please go to: http://creativecommons.org/licenses/by-nc-nd/2.5/ 


\title{
PERFORMANCE CHARACTERISATION OF PHOTOVOLTAIC MODULES
}

\author{
Ralph Gottschalg \\ Centre for Renewable Energy Systems Technology (CREST), Department of Electronic and Electrical Engineering, \\ Loughborough University, Loughborough, LE11 3TU, UK
}

\begin{abstract}
A review of the performance characterization of photovoltaic modules is given, that charts the progress made in the European research project 'PV-Performance' as well as other work carried out in Europe. The aim is to illustrate the measurement and prediction accuracy of energy delivery. It is shown that direct inter-comparisons of PV modules may have as much as $6.5 \%$ uncertainty in the comparability between modules and that any difference much lower than this is not a meaningful conclusion. A significant contribution to this is the determination of the rated power of the modules chosen for the inter-comparison and the lack of statistical numbers. The rated power is also important in the context of modeling the performance and thus must be as accurately as somewhat possible. It is shown that the uncertainties of the calibration laboratories are not borne out by round robin inter-comparisons and further work is needed in this field. Uncertainties for wafer-based devices are shown to be in a range of $\pm 3 \%$, while different thin film technologies may have higher uncertainties. It is shown that even simple modeling approaches are good enough to predict PV performance to within the measurement accuracy of most datasets.
\end{abstract}

\section{INTRODUCTION}

The energy yield of photovoltaic modules is gaining in importance when considering device characteristics. It is also becoming a sales argument and direct intercomparisons are frequently conducted. These measurements take a bit of time and thus predictive comparisons (energy rating) are desirable. Research in modeling accuracy is largely focused on obtaining better agreement, mostly to a single set of data. However, how accurate can any prediction really be? The boundaries for this are in the accuracy of the data used for the validation and uncertainties introduced by the environmental datasets. Thus this paper reviews the boundaries for accuracy in the data used for validation of any performance model, covering the inter-comparison of modules and power determination. This allows then an assessment of the modeling accuracy in the context of these boundaries and the assessment for of the potential for further improvements.

The majority of the data presented here was generated as part of the European Integrated Project 'PV-Performance'. Contributions to the following were generated by a number of laboratories, these are AIT (Austrian Institute of Technology, AT), CIEMAT (Centro de Investigaciones
Energéticas, Medioambientales y Tecnológicas, ES), CREST (UK), ECN (Energy Research Centre of the Netherlands, NL), H2M (Hochschule Magdeburg-Stendal, DE), ISE (Fraunhofer Institute for Solar Energy, DE), INES (National Institute for Solar Energy, FR), JRCESTI(European Solar Test Installation, I), LPVO (Laboratory of Photovoltaics and Optoelectronics, SL), SUPSI (University of Applied Sciences of Southern Switzerland), TUV (TUV-Rheinland, DE) and WrUT (Wroclaw University of Technology, PL).

\section{MODULE INTER-COMPARISONS}

The underlying data for performance modeling is the monitoring the operation of modules. In its simplest form, this is an inter-comparison of modules, which may also act as a marketing tool for companies. Often test winners are determined which are only fractions of a percent better than any of the modules in the same location. This in itself is not meaningful and the reasons for this statement are identified below. The accuracy of these data collections depends on the measurement accuracy of the system and the selection of modules, which is elaborated below.

\section{Measurement accuracy}

The measurement uncertainty depends to a large extent on the method of measurement. The two dominant approaches used in the EU are shown in Figure 1 and Figure 2.

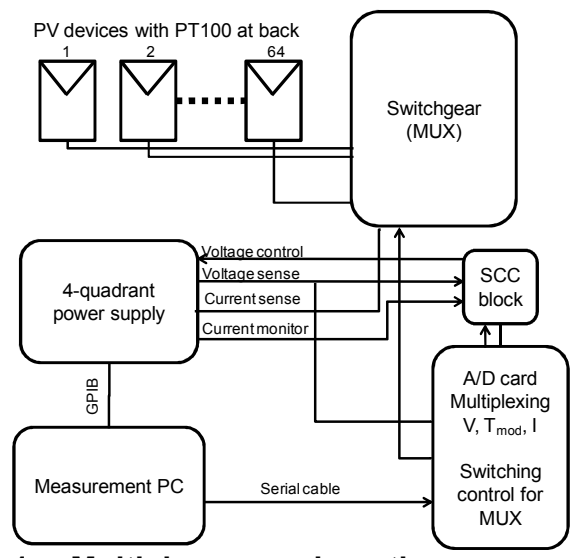

Figure 1: Multiplexer and active power supply measurement schematic.

Figure 1 depicts a system which uses a single (four quadrant) power supply to measure all devices through a multiplexer. Four wire connections are used to exclude 
the effects of slightly varying line resistances when switching through. The advantage of this system is that it is the cheapest approach to measure large number of modules. CREST, as an example, currently has more than 100 channels operational for different power levels. Other institutions using this approach are for example INES, CIEMAT, WrUT and TTU. The Joint Research Centre operates a power supply based system as well, but using a power supply per channel. This is probably the best solution but also by far the most costly.

A downside of this approach is that modules must be loaded between the I-V scans and if that is not done, one can induce e.g. very pessimistic degradation values in amorphous silicon devices [1]. This can be realized by using an appropriately sized resistance to shunt the devices between measurements.

Figure 2 depicts the approach by run by institutes such as SUPSI, where a single maximum power tracker (MPPT) with its own measurement and communication circuits is deployed for each device. Either scheme will also have the functionality to access irradiance and back of module temperatures and is linked via time-stamps to more comprehensive environmental datasets.

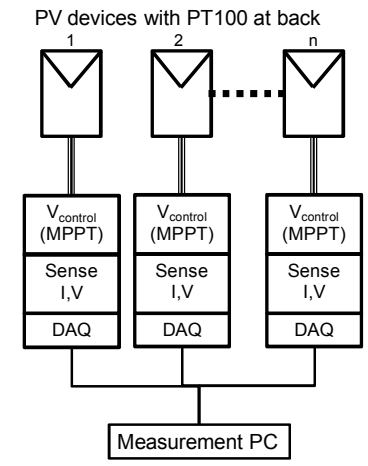

Figure 2: Maximum power point tracker based measurement schematic.

Method two has two possibilities to determine the energy yield. Some installations measure the energy yield directly from the MPPT while some carrying out an I-V sweep. The installations measuring straight from the MPPT will have an uncertainty penalty associated with the accuracy of the MPPT, as it normally does deviate slightly from the true MPPT and might not perfectly follow changing conditions. This can be estimated as an uncertainty of $0.5-1 \%$.

The inter-comparison is based on kWh. There are two possible ways to measure this, one is using a resistive network or using hall effect transducers. The latter tend to be slightly less accurate but have the enormous advantage that they protect the measurement circuit from any accidents in the circuitry and thus tend to be used in monitoring systems. The hall effect devices have nonlinearities, offsets and hysteresis. These need to be calibrated on an individual basis, i.e. not using datasheet values. When using the datasheet values, an uncertainty of about $2 \%$ will be incurred. However, one must consider the signal-dependence of the uncertainty as otherwise one quotes unrealistically small values [2]. Resistive networks would be closer to $0.2-0.5 \%$ uncertainty but one is running the risk that a failure in the circuit destroys the entire measurement system.

Typically a small number of modules is tested. Results are then sometimes in clusters, which is slightly suspicious and might indicate measurement artifacts. Care has to be taken with the location and the surroundings of the modules. Modules of differing height (e.g. a laminate mounted next to a framed module) could cause a shadow on the lower module and thus introduce apparent underperformance. Sometimes birds also like to perch on some of the modules and thus cause some persistent shading, as shown in Figure 3. Here only some of the modules were affected, while others hardly ever suffered the same fate. There is also the possibility that the albedo changes, typically by a path or some metal parts close by and thus enhancing the irradiance on a device. These effects are difficult to spot but may invalidate any intercomparison as they introduce systematic deviations.

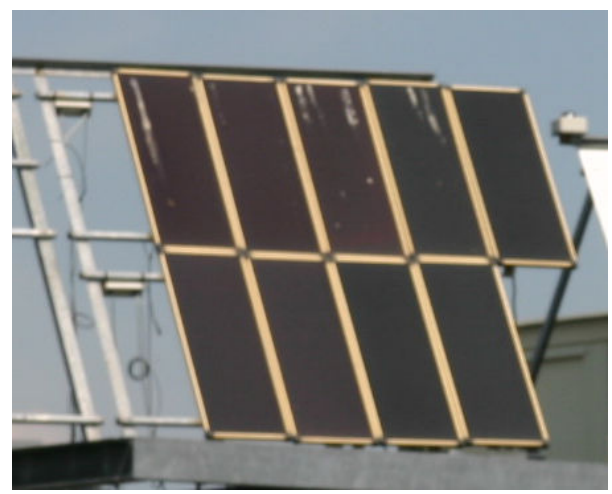

Figure 3: Natural shading events skewing the results of a module inter-comparison.

The measurement accuracy of energy can be estimated as $1-2 \%$ in well conducted measurement campaigns. $10 \%$ are easily achievable when a module is just placed outdoors and measured with an inappropriate device. The only way to circumvent the issues of artificial enhancement or waning of performance is to test statistically meaningful numbers of samples and distribute these over several positions in the test field.

\section{Module selection}

The normal outcome of inter-comparisons is the $\mathrm{kWh} / \mathrm{kWp}$. The next section will deal with the measurement of the devices, but there is also an influence of the selection of the modules. For modeling as well as inter-comparisons, this can be a significant source of uncertainty. 
The results are normalized by $\mathrm{kWp}$, but what is chosen? The options are:

1. Name plate value. The data sheet value as given by the manufacturer is chosen. The downside of this is that one needs to know where the module sits within the allowable distribution or one needs to test a number of modules that will approximate the manufacturers distribution. The uncertainty in this case is the distribution width as defined by the manufacturer plus the measurement uncertainty of the manufacturer.

2. Original power measurement. The uncertainty here is the measurement uncertainty of the power value. The downside is that it might disadvantage modules that show a deterioration in the initial phase of operation but stabilize thereafter (some monocrystalline silicon devices as well as amorphous silicon devices). On the other hand, devices that improve in the initial period of operation might be disadvantaged (e.g. some CIGS devices).

3. In service power measurements. The downside is that meta-stable materials will never have an representative value and thus can have wildly varying $\mathrm{kWh} / \mathrm{kWp}$ ratings that make any interpretation near impossible. The uncertainty here should be the measurement uncertainty plus the range of operational variation of device performance.

There is no obvious answer to this, it becomes an operators choice. However, option 1 becomes preferable if a statistically relevant number of samples is tested.

\section{Combined uncertainty}

The uncertainty for measuring the energy yield and carrying out a module inter-comparison is summarized in

\begin{tabular}{|c|c|}
\hline Uncertainty Source & Uncertainty \\
\hline Electrical measurement & $0.5-2 \%$ \\
\hline MPPT estimation & $0.5-1 \%$ \\
\hline $\begin{array}{l}\text { Variation between electrical } \\
\text { components }\end{array}$ & $1 \%$ \\
\hline $\begin{array}{ll}\text { Selection of module } \\
- & \text { Arbitrary } \\
- & \text { Initial measurement } \\
- & \text { In service measurement }\end{array}$ & $\begin{array}{l}3-5 \%+\text { measurement } \\
\text { Measurement } \\
\text { Seasonal variation }+ \text { meas }\end{array}$ \\
\hline
\end{tabular}

Thus, in order to show any meaningful difference in an inter-comparison, these differences must be larger than the uncertainty in the measurement, i.e. a minimum of $5 \%$ (assuming a measurement uncertainty of $3 \%$ ). This can only be reduced by measuring statistically relevant numbers of samples. This is also the boundary for statistically meaningful modeling accuracy.

\section{POWER MEASUREMENTS}

The power measurement in itself is a very common technique, but there may be significant uncertainties associated with this measurement influencing any performance characterisation. A summary of a questionnaire sent out by the German Solar Society (DGS), and reported by Haselhuhn [3], is summarised in Table 2. The last three laboratories may not have an ISO17025 accreditation and thus may not have gone through their uncertainty analysis in detail. It is somewhat surprising, though, that some laboratories quote the same uncertainty for thin film technologies as for wafer based devices, despite the well known complications of the testing of thin film devices.

\begin{tabular}{|l|c|c|c|c|c|}
\hline & Si & CdTe & CIGS & a-Si & m-Si \\
\hline PI Photovoltaik-Institut Berlin & $\pm 3 \%$ & $\pm 3 \%$ & $\pm 3 \%$ & $\pm 3 \%$ & $\pm 3 \%$ \\
\hline SGS Solar Test House Dresden & $\pm 3 \%$ & $\pm 3.5 \%$ & $\pm 5 \%$ & $\pm 3.5 \%$ & $\pm 3.5 \%$ \\
\hline TUV Rheinland - Cologne & $\pm 3 \%$ & $\pm 5 \%$ & $\pm 5 \%$ & $\pm 5 \%$ & $\pm 5 \%$ \\
\hline AIT Vienna & $\pm 3 \%$ & $\pm 5 \%$ & $\pm 5 \%$ & $\pm 5 \%$ & $\pm 5 \%$ \\
\hline VDE - FhG-ISE Freiburg & $\pm 3.5 \%$ & $\pm 3.5 \%$ & $\pm 3.5 \%$ & $\pm 3.5 \%$ & $\pm 3.5 \%$ \\
\hline - II - & $\pm 2.5 \%$ & $\pm 2.5 \%$ & $\pm 2.5 \%$ & $\pm 2.5 \%$ & $\pm 2.5 \%$ \\
\hline DLG, Testzentrum Technik/BM & $\pm 3.5 \%$ & - & - & $\pm 3.5 \%$ & - \\
\hline ISFH Hameln & $\pm 4 \%$ & - & - & - & - \\
\hline Photon Laboratory GmbH & $\pm 3 \%$ & $\pm 3 \%$ & $\pm 3 \%$ & $\pm 3 \%$ & $\pm 3 \%$ \\
\hline
\end{tabular}

Table 2: Return on a questionaire on claimed uncertainty of test laboratories [3].

The EU FP6 project Performance carried out two round robin (RR) inter-comparisons between 6-8 laboratories in the EU. The first of the RRs showed a good agreement with deviations from $-1.5-+2.6 \%$ [4]. However, this only covers the European area. An international round robin carried out slightly earlier showed variations of $-4.4 \%$ to $3 \%$ [5], which demonstrates that different traceability chains will be detrimental to the uncertainty in the power measurement. A second RR was carried out in 'Performance' with two additional, non-accredited laboratories included. The spread of measurements is presented in Figure 4, based on data presented in [4].

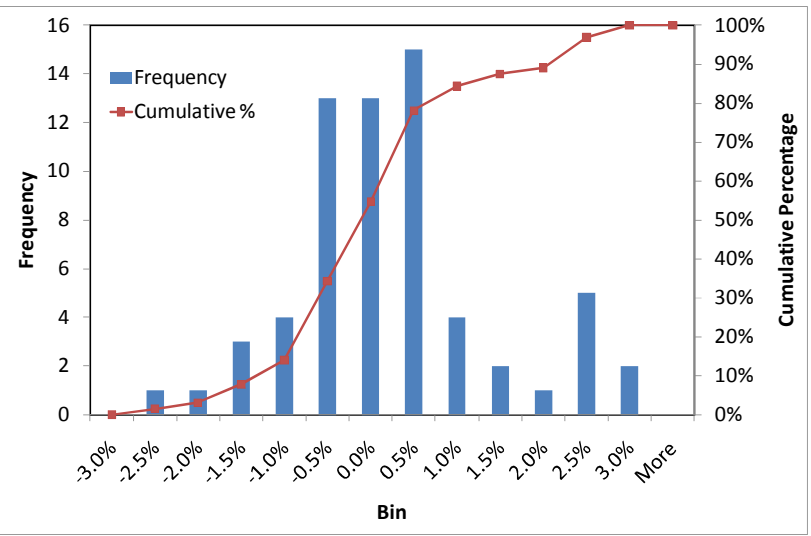

Figure 4: Frequency plot measurement results of the second round robin [4].

Surprisingly, the agreement between laboratories did not improve. However, the extremes are caused by two laboratories (one measuring high and one measuring low). These are not necessarily the two new laboratories, which indicates that the uncertainty of measurements of $3 \%$ seems to be the current limit, maybe even slightly ambitious. 
Thin films are afflicted with higher uncertainties than c-Si. In Performance's first round robin thin film uncertainties were reported as $-10 \%$ to $+9 \%$ [4]. The international round robin had slightly better results $(-8.3 \%-+8.6 \%)$ but this could be due to the different module selection in each of the round robins. The analysis showed that thin films required an adaptation of the procedures employed by the laboratories. This adaptation resulted in significant improvements, with results being ranging from $-4 \%$ to $6 \%$ deviation from the average of all measurements [4]. However, one should keep in mind that this is European laboratories only and it is unlikely that an world-wide comparison would result in the same comparability. Thus the claimed uncertainties of the laboratories appear to be rather aspirational, as they do not overlap. The biggest improvement was achieved by introducing a unified preconditioning process for the CIGS devices.

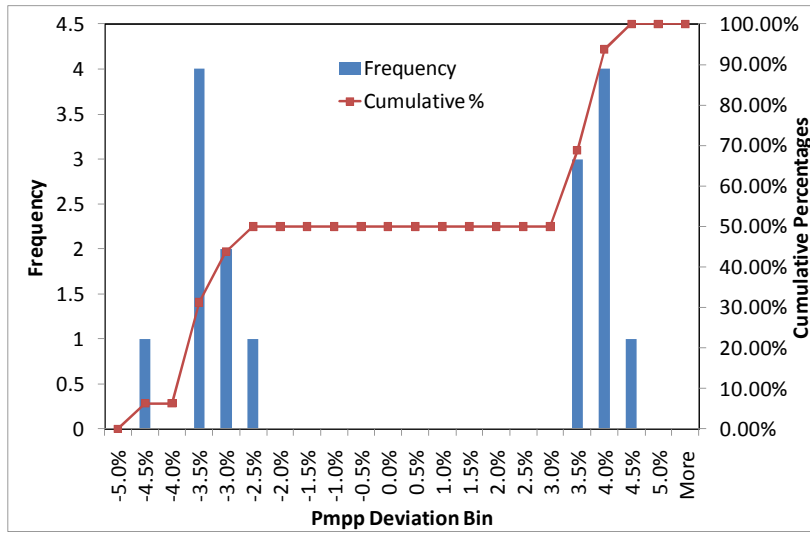

Figure 5: Round robin of four micromorph modules in four solar simulators.

The most problematic devices in all RR were multi-junction devices, as these depend strongly on the spectrum of the solar simulator. This depends also on the specifics of the devices being tested. In general the thin film silicon multijunction devices came out the worst in all round robins, as here the matching of junctions is often close to AM1.5. Slight changes in the incident spectrum can actually cause different junctions to be determining the performance. This is apparent when looking at a inter-comparison of CREST's two solar simulators (Spire 240 A, Pasan IIlb) with two industrial solar simulators (Halm and Spire 240A). The results shown in Figure 5 are curiously bimodal. The reference cell for the CREST simulators is the same, but nevertheless one of the simulators ends in the higher range and one in the lower range of the bi-modal distribution. The high measurements were both done by the Spire simulators, which would indicate that the feature is quite solar simulator specific. The good news is that here all measurements came in within less than $\pm 5 \%$ deviation of from the average. However, this depends on if the limiting junction is affected by the peaks in the spectrum of solar simulators (which is largely in the NIR range).
Energy modeling also requires measurements of non-STC conditions. This requires low light performance as well as effect of varying temperatures.

The second round robin of performance investigated these properties. Herrmann reported on these results partially [4] and further data will be released on the upcoming world conference. In the context of modeling, it is important to know the development of the overall uncertainty with different intensity levels. Measurements were taken at $100,200,800$ and $1000 \mathrm{~W} /^{2}$. The analysis of Herrmann actually showed that the level of agreement actually decreases with decreasing intensity, also with increasing standard deviation. The minimum and maximum deviations as summarised from data presented in a number of presentations are summarized in Figure 6.

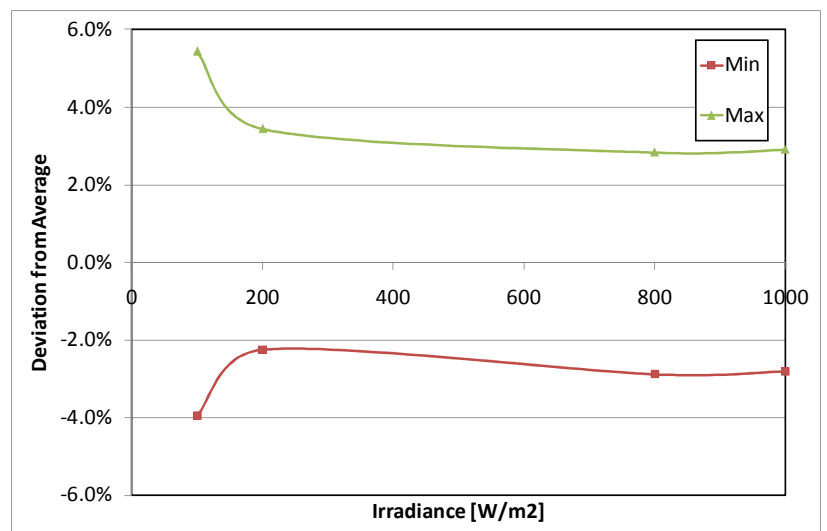

Figure 6: Variation of measurement agreement with respect to intensity variations.

The behavior of the measurements is pretty much as expected. The minimum and maximum slightly diverge for decreasing intensity, with different laboratories behaving differently. Some measuring low at STC, start measuring high with respect to the average at lower irradiances and vice versa. For very low irradiances, i.e. $100 \mathrm{~W} / \mathrm{m}^{2}$, the measurements diverge much more significantly. This is because here the method of light modification in some of the solar simulators also involves modifying the power fed to the flash bulbs, which does have a noticeable effect on the emitted spectrum.

\begin{tabular}{|l|c|c|c|c|c|}
\hline & Si & CdTe & CIGS & a-Si & m-Si \\
\hline PI Photovoltaik-Institut Berlin & $\pm 3 \%$ & $\pm 3 \%$ & $\pm 3 \%$ & $\pm 3 \%$ & $\pm 3 \%$ \\
\hline SGS Solar Test House Dresden & $\pm 3 \%$ & $\pm 3.5 \%$ & $\pm 5 \%$ & $\pm 3.5 \%$ & $\pm 3.5 \%$ \\
\hline TUV Rheinland - Cologne & $\pm 3 \%$ & $\pm 5 \%$ & $\pm 5 \%$ & $\pm 5 \%$ & $\pm 5 \%$ \\
\hline AIT Vienna & $\pm 3 \%$ & $\pm 5 \%$ & $\pm 5 \%$ & $\pm 5 \%$ & $\pm 5 \%$ \\
\hline VDE - FhG-ISE Freiburg & $\pm 3.5 \%$ & $\pm 3.5 \%$ & $\pm 3.5 \%$ & $\pm 3.5 \%$ & $\pm 3.5 \%$ \\
\hline - II - & $\pm 2.5 \%$ & $\pm 2.5 \%$ & $\pm 2.5 \%$ & $\pm 2.5 \%$ & $\pm 2.5 \%$ \\
\hline DLG, Testzentrum Technik/BM & $\pm 3.5 \%$ & - & - & - & - \\
\hline
\end{tabular}

Table 3: Claimed uncertainty of research laboratories at $200 \mathrm{~W} / \mathrm{m}^{2}$ [3].

Curiously, hardly any of the test laboratories gives a difference in their uncertainty for lower irradiances, as shown in Table 3. The claims here are for $200 \mathrm{~W} / \mathrm{m}^{2}$. The quoted uncertainties are at odds with what is shown in Figure 6 as the uncertainties partially wouldn't overlap. 
This indicates that not only is there a need for further research to increase the agreement between laboratories but also a lack of awareness of the increasing uncertainty with decreasing signal strength within the test laboratories. In light of the requirements of the energy rating standard [6], which requires the measurement or interpolation of irradiance values from $100 \mathrm{~W} / \mathrm{m}^{2}$ to $1100 \mathrm{~W} / \mathrm{m}^{2}$, it will cause a difference if measurements are taken at the extremes or closer to the centre. Clearly this needs clarification in the future.

So far the deviations between the laboratories was discussed, as these should at least overlap if the quoted values of the laboratories are correct. In terms of an error propagations, it is also relevant to know the standard deviation of the measurements. Assuming that the distributions are normal, which clearly is an oversimplification, allows the calculation of the standard deviation for the distributions reported above. The RR2 distributions for the power measurements are $1.2 \%$ for STC rising to slightly above $2.5 \%$ at $100 \mathrm{~W} / \mathrm{m}^{2}$, which means that the $95 \%$ confidence interval is twice that.

The temperature coefficient was also measured, but only by a subset of laboratories. The temperature coefficient of the power had an uncertainty of $\pm 15 \%$ [4]. This may have a very significant influence on the modeling accuracy, as temperature induced losses tend to be dominating the losses in general.

\section{ENERGY PREDICTION}

The energy prediction is affected directly by the uncertainty in the environmental dataset used as well as by the uncertainties in the device parameters used for the modeling. The section reviews the uncertainties reported in these sets before putting them into context of recent modeling round robin results. This allows identification of further room for improvement.

The key for any modeling is measured irradiance data. Secondary standard pyranometers, which are typically used for this purpose, should have less than $3 \%$ uncertainty for daily energy sums, less for longer time series. However, when bringing together a large number of sensors used by European laboratories and comparing the yield in one place, rather more significant deviations were found. The $95 \%$ confidence interval for the sensors was shown to be $3.7 \%$ deviation between the pyranometers used by the different laboratories, with all of them having up to date calibrations. The $\mathrm{k}=2$ value for the reference sensors in the same test was $4.2 \%$. $4.5 \%$ was calculated considering all sensors. This indicates that modeling cannot be more accurate than the this number if any agreement is to be statistically meaningful. This figure is for broadband irradiance and not the useful spectrum for any given device, which will vary from this. Reference cells will measure this more accurately but there are issues of stability and matching to the actual technology measured.
The second relevant environmental factor is the module temperature and how well it can be monitored. An investigation by Faiman et al [7] showed that the measurement obtained with a sensor at the back is unlikely to be better than $2^{\circ} \mathrm{C}$. This was based on the measured variations across the module in the daytime as well as in the night. This will be further influenced by the way temperature monitoring devices are attached to the modules. A yet unpublished work of Guerin de Montgareuil shows by means of a detailed balance calculation that the deviation between junction temperature and back of module temperature can be in the range of $3^{\circ} \mathrm{C}$. Put together, this would mean that the uncertainty in the measured module temperature is $3-5^{\circ} \mathrm{C}$, which equates to $1.5-2.5 \%$ power uncertainty for c-Si devices. For energy this might be less because the differences across the module as well as the difference between junction temperature and back of module temperature are reduced for lower irradiances. A good estimation would be $0.5-$ $1.5 \%$ uncertainty on energy due to temperature measurements in the incident energy.

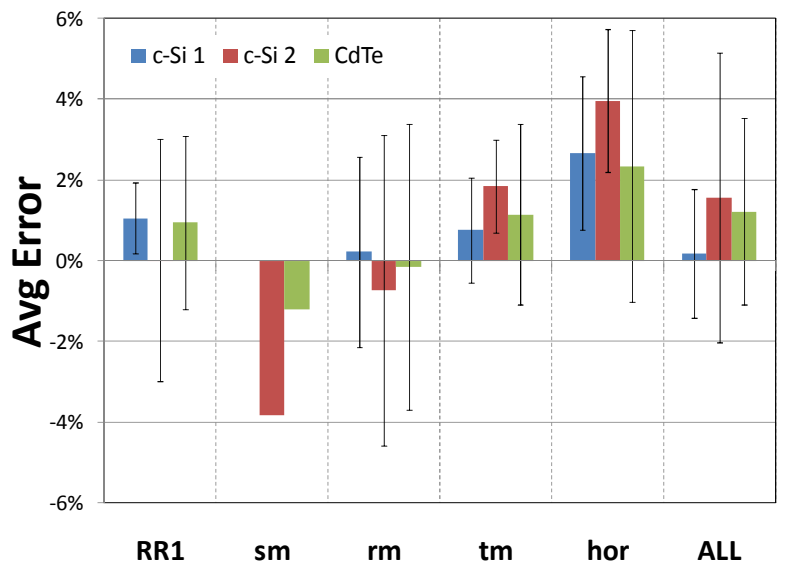

Figure 7: Development of the agreement between measurements and modeling approaches during the round robins carried out in Performance. Reproduced from [8].

The performance project had two main objectives in the modeling space. One was to evaluate the state of the art of current modeling approaches and the second was to support the energy rating standard and supply validations required. The project carried out three round robins where data was released to model the performance of devices blindly. It showed that the most challenging task was to find sets of data with a suitably low uncertainty to allow a good investigation of the accuracy of different modeling approaches. The first round robin used in plane irradiance and measured back of module temperature as an input. The agreement between measurement data and modeling results found by laboratories for this was in the range of 2$5 \%$ for different modules.

The second round robin added the translation of standard meteorological data (global horizontal irradiance, ambient 
temperature) to the data required for modeling as well as consideration of effects such as spectral variations and reflection on the module surface. The findings are summarized in Figure 7, and are discussed in more detail in [8]. The data used here was for two silicon devices one CdTe device in different locations. Translations were carried out from the base location to the target location using the methodologies of the individual laboratories. Participating laboratories were ISAAC, CREST, ECN, $\mathrm{H} 2 \mathrm{M}$, ISE, INES, JRC-ESTI and LPVO. The main difference for disagreements between the laboratories was found to be the way the laboratories derived their modeling parameters and not in the results of the calculations.

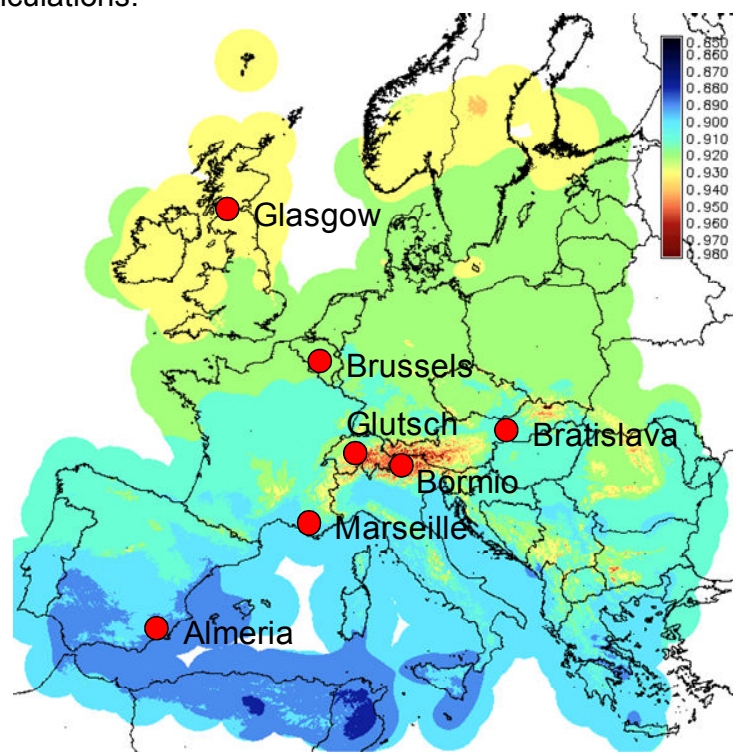

Figure 8: Overlaying standard locations as suggested by the modeling group within performance with performance maps indicating the climatic zones. Reproduced from [9].

The most significant additional deviations from the measurements were found to be the translation from the horizontal to the inclined irradiance, but within the uncertainties discussed earlier. The translation from ambient temperature to the module temperature resulted in an agreement within the uncertainty discussed earlier and thus should be considered to be as good as currently possible. This would indicate that energy prediction can only be improved if better datasets for the validation can be made available. The modeling of spectrum and reflection did not yield significant improvements. All models were all working well for sunny days but had difficulties with cloudy days.

The final aim was to look at how a possible standard dataset could look for the comparison of modules. Using standard datasets would have the advantage of removing all uncertainty in the environmental dataset, but the dataset should be accurate enough to present all performance relevant features. The approach chosen was to map the performance of different technologies, overlay this with climatic zones already derived when generating the European Solar Radiation Atlas and identifying a representative location for each climatic zone. Such a performance map (for crystalline silicon), overlaid with the suggested standard locations, is shown in Figure 8 . It is suggested to carry out performance comparisons in these locations as they are representative for wide areas. Similar approaches should be tried world-wide and a limited number of locations should be chosen to act as world-wide reference datasets.

\section{CONCLUSIONS}

The paper maps the progress in the accuracy of performance characterization through a variety of projects. The conclusion from this is that the agreement of simple models is already within the uncertainty of the typical data for the given sites, and further improvements require better monitoring data. This was shown in terms of the measurement of $\mathrm{kWh}, \mathrm{kWp}$ and irradiance measurements.

\section{ACKNOWLEDGEMENT}

The work presented was funded by a variety of sources, including the EU-FP6 programmes PV-Performance, and PV-Athlet as well as a number of national programmes funded by the EPSRC.

\section{REFERENCES}

[1] K. Astawa, et al., "Effect of Loading on Long Term Performance of Single Junction Amorphous Silicon Modules", SOLMAT, 2010, published online

[2] M. B. Strobel, et al., "Uncertainty in Photovoltaic Performance Parameters - Dependence on Location and Material", SOLMAT 93, 2009, pp. 1124-1128.

[3] R. Haselhuhn, "Modultests aus Anwendersicht", 6. Workshop Photovoltaische Modultechnik, 2009.

[4] W. Herrmann, "Leistungscharacterisierung und Vergleichbarkeit von Messergebnissen", 6. Workshop Photovoltaische Modultechnik, 2009

[5] S. Rummel, et al., "Results from the Second International Module Inter-Comparison", $4^{\text {th }}$ WCPEC.

[6] IEC, Performance Testing and Energy Rating of Terrestrial PV Modules. Part 1: Irradiance and temperature performance measurements and power rating (CDV), IEC 60904-2005 ed. Geneva: IEC, 2009.

[7] D. Faiman, et al., "Experimental Determination of the Possibility of Defining, Measuring and Predicting Cell Temperatures within a PV Module", 22nd EU-PVSEC, 2007, pp. 2527-2530.

[8] G. Friesen, et al., "Intercomparison of Different Energy Prediction Methods Within the European Project "Performance" - Results of the 2nd Round Robin," in 24th EU-PVSEC, Hamburg, 2009, pp. 3189-3197.

[9] T. Huld, et al., "Mapping the performance of PV modules, effects of module type and data averaging", Solar Energy, 84, 2010, pp. 324-338. 